\title{
An Application of the Shapley Value to Perform System Partitioning*
}

\author{
F. J. Muros ${ }^{\dagger}$, J. M. Maestre ${ }^{\dagger}$, E. Algaba ${ }^{\ddagger}$, C. Ocampo-Martinez ${ }^{\S}$ and E. F. Camacho ${ }^{\dagger}$
}

\begin{abstract}
We introduce a new method to perform the partitioning of non-centralized dynamical linear systems based on the relevance of the possible interconnections among the smallest components of the system. In particular, we analyze the importance of the interconnections using game theoretical tools, so that they can be arranged as a function of their expected contribution to the overall system performance. In addition, this method allows to identify unnecessary interconnections and highlights the most appropriate communication links facing the application of distributed control schemes. The effectiveness of the proposed method is shown at the end of this work by means of a numerical example.
\end{abstract}

\section{INTRODUCTION}

The attractive properties of non-centralized control schemes, such as their natural modularity and scalability, solve many of the challenges presented by large-scale systems. In particular, these schemes consider that the overall system is composed of several subsystems, each of them managed by a local controller or agent. In this context, we refer to decentralized control if there is no communication among the agents, i.e., the subsystems are isolated; or distributed control, in case the controllers are able to share information (see [1] for a collection of these techniques in a predictive control context).

A natural procedure before defining the agents and, in fact, necessary to determine their structure consists in the system decomposition or partitioning. This procedure, which can be developed even during the system modelling, may be based on well-established techniques reported in the literature or by physical insight, experience or intuition. However, the alternative of developing systematic methods to achieve the partitioning objective has gained importance but with only a few number of reported approaches. Since the works of Siljak [2], some considerations about the implication of the system partitioning have been analyzed in [3], relevant ideas for optimal partitioning in distributed control have been discussed in [4], and particular partitioning techniques to real case studies have been applied in water systems [5], [6], power networks [7], [8], biological systems [9] and urban traffic networks [10]. Notice that the way the system partitioning is performed might complement and/or determine

*Financial support by the COOPERA Project (DPI2013-46912-C2-1-R) and the FP7-ICT DYMASOS Proyect (under grant agreement $\mathrm{N}^{\circ}$ 611281) is gratefully acknowledged.

${ }^{\dagger}$ F. J. Muros, J. M. Maestre and E. F. Camacho are with the Department of Systems and Automation Engineering, University of Seville, Spain \{franmuros, pepemaestre, efcamacho\}eus.es

${ }^{\ddagger} \mathrm{E}$. Algaba is with the Department of Applied Mathematics II, University of Seville, Spain ealgabaeus.es

${ }^{\S}$ C. Ocampo-Martinez is with the Automatic Control Department, Universitat Politècnica de Catalunya, Institut de Robòtica i Informàtica Industrial (CSIC-UPC), Barcelona, Spain cocampo@iri.upc.edu the control strategy to be used afterwards. In fact, some partitioning criteria should be considered for the control synthesis and vice versa. It might imply the enhancement of the closed-loop performance and the computational burden associated to the resultant control strategy.

In this work, we assume that the system is initially composed of a set of indivisible pieces or atomic components that can be connected during the control system design stage. The different partitions in which the overall system is divided emerges as a result of the interconnections established among these atomic components. Once the partitions have been stated, an agent is assigned to each of them in order to achieve the satisfaction of the local control objectives. After this step, it is also interesting to consider whether some of these partitions should be grouped into different coalitions, in order to implement a distributed control algorithm. The identification of the atomic components and the agents that should be connected is the main contribution of this article. To this end, game theoretical tools are used. In particular, the possible connections that can be established are considered as the players of a cooperative game. In order to distribute the benefit among these players, we choose the well-known payoff rule called Shapley value [11] as a solution concept of the game. In this way, a method to establish the expected utility for the interconnections is developed based on the Shapley value in order to determine the system partitioning and highlight unnecessary links and potential alliances for the application of distributed control schemes.

It must be noticed that this line of research is closely related with the so-called coalitional control, which considers explicitly that the interactions among the agents evolve dynamically with time and takes advantage of this fact in order to reduce the communication burden without compromising the system performance. Some examples can be found in [12], which analyzes several hierarchical control structures to implement the most appropriate one; [13], [14], where the control scheme enables or disables links depending on their contribution to the overall system objectives; or [15], where a bottom-up coalition formation algorithm on a water system is proposed. Recently in [16], [17] some game theorybased refinements of [13], [14] are given in order to impose additional constraints to these links.

The remainder of the paper is organized as follows. In Section II, a preliminary setting is provided. In Section III, metrics to measure the importance of the links are presented and the proposed partitioning algorithm is given in Section IV. In Section V, a simple numerical example is used to illustrate the proposed approach. Finally, conclusions and comments about future research are drawn in Section VI. 


\section{PROBLEM DESCRIPTION}

In this section, we first present the model used to represent the system dynamics. Next, we define the control goal. Finally, some tools from cooperative game theory are applied to the control scheme.

\section{A. System Description}

Consider the class of distributed linear systems composed of $\mathcal{N}=\{1,2, \ldots,|\mathcal{N}|\}$ interconnected atomic components. The dynamics of the atomic component $i \in \mathcal{N}$ can be described mathematically as

$$
\begin{gathered}
\mathbf{x}_{i}(k+1)=\mathbf{A}_{i i} \mathbf{x}_{i}(k)+\mathbf{B}_{i i} \mathbf{u}_{i}(k)+\mathbf{d}_{i}(k), \\
\mathbf{d}_{i}(k)=\sum_{j \neq i}\left[\mathbf{A}_{i j} \mathbf{x}_{j}(k)+\mathbf{B}_{i j} \mathbf{u}_{j}(k)\right],
\end{gathered}
$$

where $\mathbf{x}_{i}(k) \in \mathbb{R}^{n_{\mathbf{x}_{i}}}$ is the state of the atomic component $i, \mathbf{u}_{i}(k) \in \mathbb{R}^{n_{\mathrm{u}_{i}}}$ is its corresponding input, and $\mathbf{A}_{i j} \in$ $\mathbb{R}^{n_{\mathrm{x}_{i}} \times n_{\mathrm{x}_{j}}}, \mathbf{B}_{i j} \in \mathbb{R}^{n_{\mathrm{x}_{i}} \times n_{\mathrm{u}_{j}}}$ are, respectively, the state transition and the input-to-state matrices. The influence of the neighbors in the atomic component $i$ is given by $\mathbf{d}_{i}(k)$.

The overall system can be described as

$$
\mathbf{x}_{\mathcal{N}}(k+1)=\mathbf{A}_{\mathcal{N}} \mathbf{x}_{\mathcal{N}}(k)+\mathbf{B}_{\mathcal{N}} \mathbf{u}_{\mathcal{N}}(k),
$$

where $\mathbf{x}_{\mathcal{N}}(k)=\left[\mathbf{x}_{1}(k), \ldots, \mathbf{x}_{|\mathcal{N}|}(k)\right]^{\mathrm{T}} \in \mathbb{R}^{n_{\mathbf{x}_{\mathcal{N}}}}, \mathbf{u}_{\mathcal{N}}(k)=$ $\left[\mathbf{u}_{1}(k), \ldots, \mathbf{u}_{|\mathcal{N}|}(k)\right]^{\mathrm{T}} \in \mathbb{R}^{n_{\mathbf{u}_{\mathcal{N}}}}$ are, respectively, the global state and the global input vectors, and $\mathbf{A}_{\mathcal{N}}=\left[\mathbf{A}_{i j}\right]_{i, j \in \mathcal{N}} \in$ $\mathbb{R}^{n_{\mathrm{x}_{\mathcal{N}}} \times n_{\mathrm{x}_{\mathcal{N}}}}, \mathbf{B}_{\mathcal{N}}=\left[\mathbf{B}_{i j}\right]_{i, j \in \mathcal{N}} \in \mathbb{R}^{n_{\mathrm{x}_{\mathcal{N}}} \times n_{\mathrm{u}_{\mathcal{N}}}}$ are the state transition and the input-to-state global matrices.

The set of atomic components and all the possible interconnections among them can be described by a complete undirected graph $(\mathcal{N}, \mathcal{E})$, with $\mathcal{E}=\mathcal{N} \times \mathcal{N}$, which verifies

$$
|\mathcal{E}|=\frac{|\mathcal{N}||\mathcal{N}-1|}{2},
$$

where we will assume a cost $c \in \mathbb{R}^{+} \backslash\{0\}$ for each possible connection $l \in \mathcal{E}$.

Definition 1 We call interconnection topology, denoted by $\Lambda(k)$, each of the different configurations for the partitions depending on the established connections among the atomic components in a time step $k$. Notice that there are $2^{|\mathcal{E}|}$ possible topologies that can be formed from the graph $(\mathcal{N}, \mathcal{E})$, which will be denoted as $\Lambda_{0}(k), \Lambda_{1}(k), \ldots, \Lambda_{2^{|\mathcal{E}|-1}}(k)$.

The key is to determine the topology that establishes the more appropriate connections among the atomic components in order to define the partitions for the system. In this way, we consider the following set of agents to govern each of these partitions: $\mathcal{M}=\left\{a_{1}, a_{2}, \ldots, a_{|\mathcal{M}|}\right\}$, where $|\mathcal{M}| \leq|\mathcal{N}|$.

Example 1 Consider the 5 atomic components shown in Figure $1 a$, where we represent the 10 potential interconnections among them in roman letters. In this way, we have $2^{10}$ possible configurations or topologies represented in Table I. For instance, for the particular case of the topology $\Lambda_{7}(k)$, we only have enabled the link VII and this configuration provides the four partitions represented in Figure $1 b$.

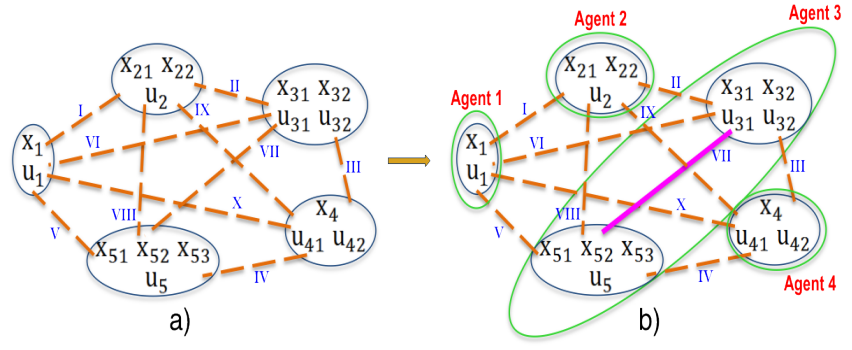

Fig. 1. Interconnection topology $\Lambda_{7}(k)$ in Example 1

TABLE I

INTERCONNECTION TOPOLOGIES AND NUMBER OF PARTITIONS

\begin{tabular}{|c|c|c|c|}
\hline \multicolumn{2}{|c|}{ Topologies } & Atomic components & Partitions \\
\hline \hline$\Lambda_{0}(k)$ & $\{\emptyset\}$ & $\{1\},\{2\},\{3\},\{4\},\{5\}$ & 5 \\
$\Lambda_{1}(k)$ & $\{I\}$ & $\{1,2\},\{3\},\{4\},\{5\}$ & 4 \\
$\vdots$ & $\vdots$ & $\vdots$ & $\vdots$ \\
$\Lambda_{7}(k)$ & $\{I\}$ & $\{1\},\{2\},\{3,5\},\{4\}$ & 4 \\
$\vdots$ & $\vdots$ & $\vdots$ & $\vdots$ \\
$\Lambda_{20}(k)$ & $\{I I, I I I\}$ & $\{1\},\{2,3,4\},\{5\}$ & 3 \\
$\vdots$ & $\vdots$ & $\vdots$ & $\vdots$ \\
$\Lambda_{100}(k)$ & $\{I I, I V, V I\}$ & $\{1,2,3\},\{4,5\}$ & 2 \\
$\vdots$ & $\vdots$ & $\vdots$ & $\vdots$ \\
$\Lambda_{1023}(k)$ & $\mathcal{E}$ & $\mathcal{N}$ & 1 \\
\hline
\end{tabular}

Once the topology is fixed and the agents are established, it is relevant to study what links are unnecessary or which ones can be useful for distributed control purposes. An algorithm for defining the partitions and removing the unnecessary links among them will be presented in Section IV.

\section{B. Partitioning Goal}

In this section, we present the goal that is pursued in the choice of the interconnections among the pieces in which the system is divided. In this way, we assume that the overall cost of regulating the system to the origin for a given topology $\Lambda(k)$ is formulated by

$$
J(k)=\overbrace{\left(\sum_{j=0}^{\infty}\left[\mathbf{x}_{\mathcal{N}}^{\mathrm{T}}(k+j) \mathbf{Q}_{\mathcal{N}} \mathbf{x}_{\mathcal{N}}(k+j)+\mathbf{u}_{\mathcal{N}}^{\mathrm{T}}(k+j) \mathbf{R}_{\mathcal{N}} \mathbf{u}_{\mathcal{N}}(k+j)\right]\right)}^{J_{s}(k)}+\overbrace{c|\Lambda(k)|}^{J_{c}(k)},
$$

with $J_{s}(k), J_{c}(k) \in \mathbb{R}^{+}$being, respectively, the cost-to-go

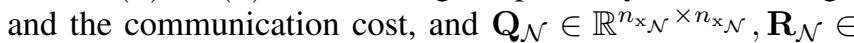
$\mathbb{R}^{n_{\mathrm{u}_{\mathcal{N}}} \times n_{\mathrm{u}_{\mathcal{N}}}}$ positive definite and semi-definite weighting matrices, respectively ${ }^{1}$. To this end, the following topologydependant feedback control law is applied:

$$
\mathbf{u}_{\mathcal{N}}=\mathbf{K}_{\Lambda} \mathbf{x}_{\mathcal{N}}
$$

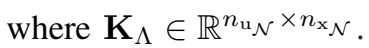

The problem of minimizing (4) is difficult to solve because it is not convex [18]. In order to obtain at least a suboptimal solution, we assume that we can find a positive definite matrix $\mathbf{P}_{\Lambda} \in \mathbb{R}^{n_{\mathrm{x}_{\mathcal{N}}} \times n_{\mathrm{x}_{\mathcal{N}}}}$ that provides a Lyapunov function $f\left(\Lambda, \mathbf{x}_{\mathcal{N}}\right)=\mathbf{x}_{\mathcal{N}}^{\mathrm{T}} \mathbf{P}_{\Lambda} \mathbf{x}_{\mathcal{N}}$ of the closed-loop system (when the control law (5) is applied), and it also satisfies, $\forall \mathbf{x}_{\mathcal{N}}, \Lambda$,

$$
\mathbf{x}_{\mathcal{N}}^{\mathrm{T}} \mathbf{P}_{\Lambda} \mathbf{x}_{\mathcal{N}} \geq J_{s}
$$

\footnotetext{
${ }^{1}$ In the following, we will omit the dependence of states, inputs and costs on time step $k$ for the sake of clarity.
} 
Notice that $\mathbf{K}_{\Lambda}$ and $\mathbf{P}_{\Lambda}$ are connected by [13]

$$
\overbrace{\mathbf{x}_{\mathcal{N}}^{\mathrm{T}+} \mathbf{P}_{\Lambda} \mathbf{x}_{\mathcal{N}}^{+}}^{\geq J_{s}^{+}}+\overbrace{\mathbf{x}_{\mathcal{N}}^{\mathrm{T}} \mathbf{Q}_{\mathcal{N}} \mathbf{x}_{\mathcal{N}}+\mathbf{x}_{\mathcal{N}}^{\mathrm{T}} \mathbf{K}_{\Lambda}^{\mathrm{T}} \mathbf{R}_{\mathcal{N}} \mathbf{K}_{\Lambda} \mathbf{x}_{\mathcal{N}}}^{\text {stage cost }} \leq \overbrace{\mathbf{x}_{\mathcal{N}}^{\mathrm{T}} \mathbf{P}_{\Lambda} \mathbf{x}_{\mathcal{N}}}^{\geq J_{s}},
$$

where superindex + refers to dependence on $k+1$. Hence, different $\mathbf{K}_{\Lambda}$ and $\mathbf{P}_{\Lambda}$ must be found for each topology $\Lambda$. In this work, we will obtain these matrices by considering linear matrix inequalities (LMIs) and the Matlab ${ }^{\circledR}$ LMI Control Toolbox [19] to solve them. In this way, it is possible to rewrite (7) into the following LMI (see [13]):

$$
\begin{gathered}
{\left[\begin{array}{cccc}
\mathbf{W}_{\Lambda} & \mathbf{W}_{\Lambda} \mathbf{A}_{\mathcal{N}}^{\mathrm{T}}+\mathbf{Y}_{\Lambda}^{\mathrm{T}} \mathbf{B}_{\mathcal{N}}^{\mathrm{T}} & \mathbf{W}_{\Lambda} \mathbf{Q}_{\mathcal{N}}^{1 / 2} & \mathbf{Y}_{\Lambda}^{\mathrm{T}} \mathbf{R}_{\mathcal{N}}^{1 / 2} \\
\mathbf{A}_{\mathcal{N}} \mathbf{W}_{\Lambda}+\mathbf{B}_{\mathcal{N}} \mathbf{Y}_{\Lambda} & \mathbf{W}_{\Lambda} & 0 & 0 \\
\mathbf{Q}_{\mathcal{N}}^{1 / 2} \mathbf{W}_{\Lambda} & 0 & \mathbf{I} & 0 \\
\mathbf{R}_{\mathcal{N}}^{1 / 2} \mathbf{Y}_{\Lambda} & 0 & 0 & \mathbf{I}
\end{array}\right]>0} \\
\\
i \leftrightarrow j \Longrightarrow\left\{\begin{array}{c}
\mathbf{Y}_{\Lambda}^{i j}=\mathbf{Y}_{\Lambda}^{j i}=0 \\
\mathbf{W}_{\Lambda}^{i j}=\mathbf{W}_{\Lambda}^{j i}=0
\end{array}\right.
\end{gathered}
$$

where $\mathbf{W}_{\Lambda}=\mathbf{P}_{\Lambda}^{-1}$ and $\mathbf{Y}_{\Lambda}=\mathbf{K}_{\Lambda} \mathbf{P}_{\Lambda}^{-1}$ are the decision variables, matrix I denotes the identity matrix of suitable dimensions, and ( $8 \mathrm{~b}$ ) considers the communication constraints imposed by the particular topology $\Lambda$ (see [16], [17] for further details).

Note that if there is a feasible solution of the LMI in (8), it could be possible to find a matrix $\mathbf{P}_{\Lambda}$ that verifies (6). In this case, and according to [13], we can define, for each topology $\Lambda$, the following upper bound on the cost function $J$, whose minimization can be assumed as the partitioning goal:

$$
r^{v}\left(\Lambda, \mathbf{x}_{\mathcal{N}}\right)=\mathbf{x}_{\mathcal{N}}^{\mathrm{T}} \mathbf{P}_{\Lambda} \mathbf{x}_{\mathcal{N}}+c|\Lambda|
$$

\section{Game Theoretical Perspective}

The key idea proposed in [13], [14], [16], [17] is to interpret (9) as the characteristic function of a cooperative game in which the set of interconnections $l \in \mathcal{E}$ is the set of players. In this context, it is possible to use the pair $\left(\mathcal{E}, \boldsymbol{r}^{v}\right)$ to define a cooperative game with transferable utility. Once the game is obtained, the cost of each link is computed by means of an allocation rule. Note that useful links will be related to lower costs. In particular, we will apply the Shapley value [11], which assigns to the game $\left(\mathcal{E}, \boldsymbol{r}^{\boldsymbol{v}}\right)$ the vector $\phi\left(\mathcal{E}, \boldsymbol{r}^{\boldsymbol{v}}\right)$ that is defined $\forall l \in \mathcal{E}$ as

$\phi_{l}\left(\mathcal{E}, \boldsymbol{r}^{v}\right)=\sum_{\Lambda \subseteq \mathcal{E}, l \notin \Lambda} \frac{|\Lambda| !(|\mathcal{E}|-|\Lambda|-1) !}{|\mathcal{E}| !}\left[r^{v}\left(\Lambda \cup\{l\}, \mathbf{x}_{\mathcal{N}}\right)-r^{v}\left(\Lambda, \mathbf{x}_{\mathcal{N}}\right)\right]$.

Therefore, through the Shapley value, each connection gets a weighted average of its contribution to the different partitions the system can be divided. In addition, given a linkgame $\left(\mathcal{E}, \boldsymbol{r}^{v}\right)$, the Shapley value $\phi\left(\mathcal{E}, \boldsymbol{r}^{v}\right)$ can be represented by the Shapley standard matrix $\mathbf{M} \in \mathbb{R}^{|\mathcal{E}| \times 2^{|\mathcal{E}|}}$ as [20]

$$
\phi\left(\mathcal{E}, \boldsymbol{r}^{\boldsymbol{v}}\right)=\left[\begin{array}{c}
\phi_{I} \\
\phi_{I I} \\
\vdots \\
\phi_{|\mathcal{E}|}
\end{array}\right]=\mathbf{M} \boldsymbol{r}^{\boldsymbol{v}}
$$

where each element of $\mathbf{M}$, denoted by $m_{l \Lambda}$, is defined as

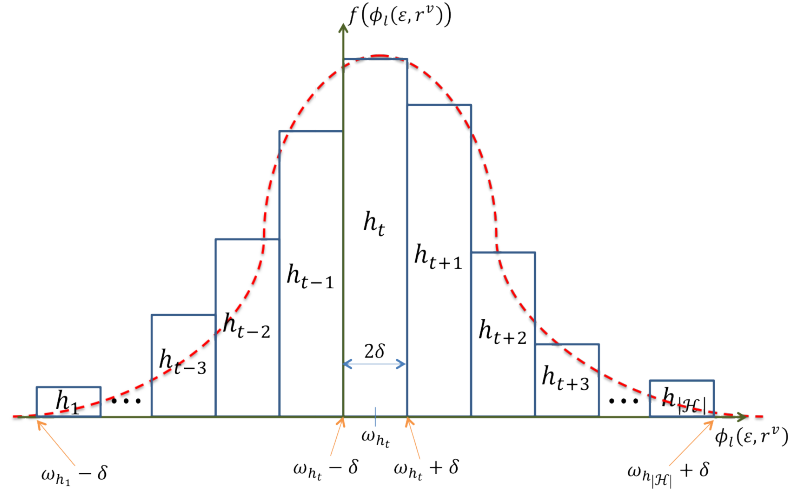

Fig. 2. Probability density function for the Shapley value

$$
m_{l \Lambda}= \begin{cases}\frac{(|\Lambda|-1) !(|\mathcal{E}|-|\Lambda|) !}{|\mathcal{E}| !} & l \in \Lambda, \\ -\frac{|\Lambda| !(|\mathcal{E}|-|\Lambda|-1) !}{|\mathcal{E}| !} & l \notin \Lambda .\end{cases}
$$

\section{PARTITIONING METRICS}

Next, we propose metrics to find out which interconnections are more relevant and which ones are expendable by means of statistical parameters [21].

\section{A. The Shapley Value Probability Density Function}

Given a set of interconnections among atomic components described by the graph $(\mathcal{N}, \mathcal{E})$, we want to obtain a mapping for the possible Shapley values that each connection $l \in \mathcal{E}$ can achieve. In this way, we assume that the set of states $\mathbf{x}_{\mathcal{N}}$ are confined into a certain domain, i.e.,

$$
\mathbf{x}_{\mathcal{N}} \in \mathcal{X}_{\mathcal{N}}, \quad \mathcal{X}_{\mathcal{N}} \subseteq \mathbb{R}^{n_{\mathrm{x}_{\mathcal{N}}}}
$$

Note that, the cost defined by (9), and consequently the Shapley value (11) will both take values into intervals of real numbers, i.e.,

$$
\begin{gathered}
r^{v}\left(\Lambda, \mathbf{x}_{\mathcal{N}}\right) \in\left[r_{\min }^{v}, r_{\max }^{v}\right] \\
\phi_{l}\left(\mathcal{E}, \boldsymbol{r}^{\boldsymbol{v}}\right) \in\left[\phi_{l}^{\text {min }}, \phi_{l}^{\max }\right] .
\end{gathered}
$$

The key idea is to obtain a way to arrange and compare the links according to their relevance from a control performance perspective. This ranking of links has to be independent from a given state $\mathbf{x}_{\mathcal{N}}$. To this end, the cost of the system is evaluated by using (9) for a large number of random state samples so that general results are obtained regarding the Shapley value of the interconnections. In this way, the following procedure is proposed:

\section{Procedure}

Let $L \in \mathbb{N}^{+} \backslash\{0\}$ be a number of samples. Let

$$
\mathcal{H}=\left\{h_{1}, h_{2}, \ldots, h_{|\mathcal{H}|}\right\}, \forall h \in \mathcal{H} \quad h:=\left[\omega_{h}-\delta, \omega_{h}+\delta\right)
$$

be also a set of consecutive intervals, with $\omega_{h} \in \mathbb{R}, \delta \in$ $\mathbb{R}^{+} \backslash\{0\}$, and satisfying $\omega_{h}+\delta=\omega_{h+1}-\delta, \forall h$ (see Figure 2).

Finally, let $\mathbf{K}_{\Lambda}=\mathbf{Y}_{\Lambda} \mathbf{W}_{\Lambda}^{-1}$ and $\mathbf{P}_{\Lambda}=\mathbf{W}_{\Lambda}^{-1}$ be the corresponding solution of the optimization problem, $\forall \Lambda$

$$
\max _{\mathbf{W}_{\Lambda}, \mathbf{Y}_{\Lambda}} \operatorname{Tr}\left(\mathbf{W}_{\Lambda}\right),
$$

subject to (8). 
We define an iteration index $s$ and a set of counter variables $r_{h}$ associated to each interval $h$. We start with $s=1$ and $r_{h}=0, \forall h \in \mathcal{H}$, and we do the following:

1) Take a random sample for the global state $\mathrm{x}_{\mathcal{N}} \in \mathcal{X}_{\mathcal{N}}$.

2) Obtain $r^{v}\left(\Lambda, \mathbf{x}_{\mathcal{N}}\right), \forall \Lambda \subseteq \mathcal{E}$ and $\phi_{l}\left(\mathcal{E}, \boldsymbol{r}^{\boldsymbol{v}}\right), \forall l \in \mathcal{E}$ by using (9) and (11), respectively.

3) For each interval $h \in \mathcal{H}$ and for each link $l \in \mathcal{E}$

$$
\text { if } \phi_{l}\left(\mathcal{E}, \boldsymbol{r}^{\boldsymbol{v}}\right) \in h \text { then } r_{h}=r_{h}+1 \text {. }
$$

4) Make $s=s+1$ and go to Step 1 while $s<L$.

After the application of the aforementioned procedure, if we normalize the resulting function by $\frac{L}{2 \delta}$, we get a discretization for the probability density function $f\left(\phi_{l}\left(\mathcal{E}, \boldsymbol{r}^{\boldsymbol{v}}\right)\right)$ of each Shapley value $\phi_{l}\left(\mathcal{E}, \boldsymbol{r}^{\boldsymbol{v}}\right)$ (performed in blue in Figure 2), which is a numerical approximation of the real shape for the density function (represented in dashed red).

\section{B. Characteristic Index}

Once we have an estimation of the Shapley value probability density function, we need to arrange the links as a function of their relevance. Hence, it is necessary to establish metrics that do not depend on the dynamics of a specific system. In this way, we propose the following normalized index, with $l \in \mathcal{E}$

$$
\overline{\mu_{l}}=\frac{\mu_{l}}{\phi_{l}^{\max }-\phi_{l}^{\min }},
$$

where $\mu_{l}$ is the mean parameter for probability density functions, defined as [21]

$$
\mu_{l}=\int_{-\infty}^{\infty} \phi_{l}\left(\mathcal{E}, \boldsymbol{r}^{\boldsymbol{v}}\right) f\left(\phi_{l}\left(\mathcal{E}, \boldsymbol{r}^{\boldsymbol{v}}\right)\right) d \phi_{l}
$$

In this sense, we consider that a link will be more useful as lower mean it has.

Remark 1 It would be possible to extend these results by considering non-equiprobable states, and by working with percentiles -or other parameters such as the variation coeficient-instead the mean (see [21]).

\section{PARTITIONING ALGORITHM}

First, we group the atomic components connected by the graph $(\mathcal{N}, \mathcal{E})$ into partitions. Next we remove redundant and expensive links. The steps are presented here and an algorithm is given at the end of this section.

\section{A. Establishing Partitions}

With the purpose of defining the best connections among the atomic components, we introduce the set $\mathcal{E}_{c} \subseteq \mathcal{E}$ that will include all the links that are enough useful to be fixed. Each link that belongs to this set must satisfy the following:

$$
l \in \mathcal{E}_{c} \longleftrightarrow \overline{\mu_{l}}<\mathcal{L}_{c},
$$

with $\mathcal{L}_{c} \in \mathbb{R}$ a constant threshold.

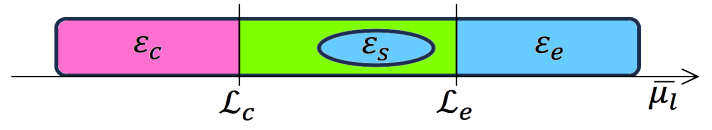

Fig. 3. Partitioning algorithm diagram

That is, the criterion will be to join the atomic components by using links $l \in \mathcal{E}_{c}$. We assume that these links define the way in which the different subsystems or partitions are built. In other words, links $l \in \mathcal{E}_{c}$ are assumed to be part of the system structure once they have been set. Hence, from now on, we will work with partitions, and consequently with agents $a \in \mathcal{M}$

\section{B. Redundant Links Removal}

If the set $\mathcal{E}_{c} \neq\{\emptyset\}$, there will be pairs of agents with more than one direct connection between them. See for example the links $I I I$ and $I V$ that connect both the agents 3 and 4 in the example shown in Figure 1b.

Given two agents $a_{p}, a_{q} \in \mathcal{M}$, we denote the set of direct links between them as $\mathcal{E}_{p q}=\left\{l_{p q}^{1}, l_{p q}^{2}, \ldots, l_{p q}^{n}\right\}$, with $\mathcal{E}_{p q} \subseteq \mathcal{E} \backslash \mathcal{E}_{c}$. From all these links, we will choose the one that satisfies

$$
l_{p q}^{*} \in \mathcal{E}_{p q} \text { such that } \overline{\mu_{l_{p q}^{*}}}=\min _{i}\left\{\overline{\mu_{l_{p q}^{i}}}\right\}, l_{p q}^{i} \in \mathcal{E}_{p q} .
$$

Therefore, redundant links $l \in \mathcal{E}_{p q} \backslash\left\{l_{p q}^{*}\right\}$ can be rejected. Let us generalize (21) for all the possible pairs of agents $a_{p}, a_{q} \in \mathcal{M}$ in order to define the following set of redundant links:

$$
\mathcal{E}_{s}=\bigcup_{a_{p}, a_{q} \in \mathcal{M}}\left(\mathcal{E}_{p q} \backslash\left\{l_{p q}^{*}\right\}\right) .
$$

Finally, note that the following is trivially verified:

$$
\mathcal{E}_{c}=\{\emptyset\} \rightarrow \mathcal{E}_{s}=\{\emptyset\} .
$$

\section{Low-performance Links Removal}

Once we have defined the partitions and eliminated the redundant links, it is possible to calculate whether there are any other links that are too expensive to be considered. In this way, we introduce the set $\mathcal{E}_{e} \subseteq \mathcal{E} \backslash\left(\mathcal{E}_{c} \cup \mathcal{E}_{s}\right)$ that will include all the links that satisfy the following:

$$
l \in \mathcal{E}_{e} \longleftrightarrow \overline{\mu_{l}}>\mathcal{L}_{e}
$$

with $\mathcal{L}_{e} \in \mathbb{R}$ a constant threshold.

In other words, we will consider that links $l \in \mathcal{E}_{e}$ are permanently disconnected since they are expensive enough for the system.

\section{Design Algorithm}

The following algorithm provides a method for performing the system partitioning. In addition, it supplies information regarding the relevance of the links, which is useful in order to establish a reduced number of communication links among the different agents. Consequently, it may simplify the implementation of distributed control schemes. A diagram that summarizes the following partitioning method is given in Figure 3. 


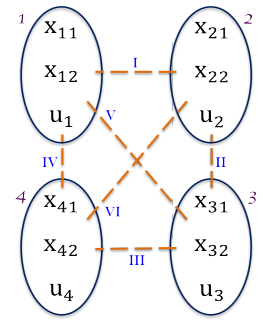

Fig. 4. Simulation example: initial atomic components

\section{Algorithm}

Let $(\mathcal{N}, \mathcal{E})$ be an undirected graph that describes a set $\mathcal{N}$ of atomic components connected by links $l \in \mathcal{E}$. Let $\mathcal{L}_{c}, \mathcal{L}_{e} \in \mathbb{R}$ be given thresholds. Then,

1) By using the procedure presented in Subsection III-A and (18), calculate $\overline{\mu_{l}}, \forall l \in \mathcal{E}$.

2) Apply (20) in order to define the set $\mathcal{E}_{c}$. Links $l \in \mathcal{E}_{c}$ will be preserved and will define agents $a \in \mathcal{M}$.

3) Disconnect all redundant links $l \in \mathcal{E}_{s}$, according to (22).

4) Apply (24) in order to define the set $\mathcal{E}_{e}$. Lowperformance links $l \in \mathcal{E}_{e}$ will be also discarded.

Therefore, the configuration of the system will be described by the following network:

$$
\left(\mathcal{M}, \mathcal{E} \backslash\left(\mathcal{E}_{c} \cup \mathcal{E}_{s} \cup \mathcal{E}_{e}\right)\right),
$$

and different control techniques may be applied at this point.

Remark 2 Links $l \in \mathcal{E}_{c}$ (represented in pink in the diagram of Figure 3) become a definitive part of the system structure and define the partitions. Links $l \in\left(\mathcal{E}_{s} \cup \mathcal{E}_{e}\right)$ (performed in blue) will be permanently disabled. The rest of the links (represented in green) connect partitions and will be enabled or disabled depending on the needs of the particular control scheme implemented by the agents.

\section{SIMULATION RESULTS}

In this section, we study the case shown in Figure 4, whose atomic components and interconnections are described by the graph $(\mathcal{N}, \mathcal{E})$, with $\mathcal{N}=\{1,2,3,4\}$ and $\mathcal{E}=\{I, I I, I I I, I V, V, V I\}$. The matrices that define the subsystem's dynamics are

$$
\begin{gathered}
\mathbf{A}_{11}=\left[\begin{array}{ll}
1 & 0.8 \\
0 & 0.7
\end{array}\right], \mathbf{A}_{22}=\left[\begin{array}{cc}
1 & 0.9 \\
0 & -2.5
\end{array}\right], \mathbf{A}_{33}=\left[\begin{array}{cc}
1 & -2 \\
0 & 3
\end{array}\right], \mathbf{A}_{44}=\left[\begin{array}{ll}
1 & 2.2 \\
0 & 0.5
\end{array}\right], \\
\mathbf{B}_{i i}=\left[\begin{array}{l}
0 \\
1
\end{array}\right], \quad \mathbf{A}_{i j}=\left[\begin{array}{ll}
0 & 0 \\
0 & 0
\end{array}\right], \mathbf{B}_{i j}=\left[\begin{array}{c}
0 \\
0.15
\end{array}\right], i \neq j,
\end{gathered}
$$

where $\mathbf{x}_{i} \in \mathbb{R}^{2}$ and $\mathbf{u}_{i} \in \mathbb{R}$ are, respectively, the states and the input of each atomic component $i \in \mathcal{N}$. The cost-to-go $J_{s}$ is defined by matrices $\mathbf{Q}=\mathbf{I} \in \mathbb{R}^{8 \times 8}$ and $\mathbf{R}=\mathbf{I} \in \mathbb{R}^{4 \times 4}$. We also suppose a cost $c=0.5$ per enabled connection.

For the configuration of this system, we will consider the following thresholds:

$$
\begin{gathered}
\mathcal{L}_{c}=-0.2, \\
\mathcal{L}_{e}=-0.1 .
\end{gathered}
$$

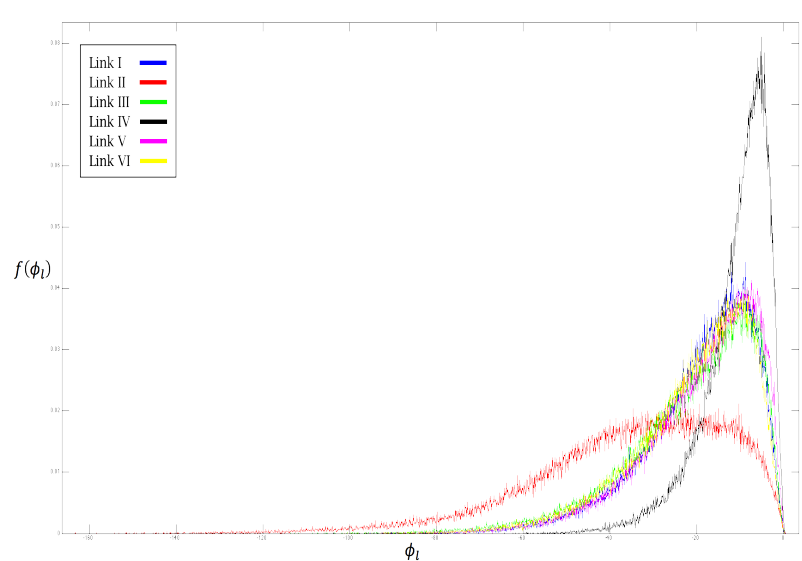

Fig. 5. Probability density function for the Shapley values

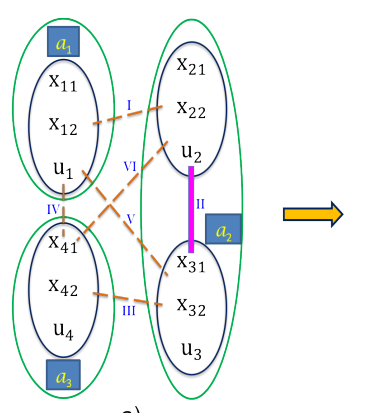

a)

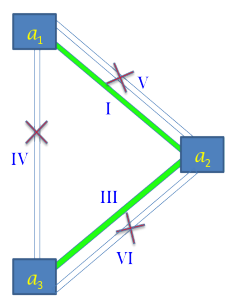

b)
Fig. 6. System partitioning

If we take $L=10^{5}$ and $\delta=0.05$ in the procedure of Subsection III-A, the resulting probability density function for the Shapley value of each link is depicted in Figure 5. The corresponding normalized means are

$$
\begin{aligned}
& \overline{\mu_{I}}=-0.1164, \quad \overline{\mu_{I I}}=-0.2106, \quad \overline{\mu_{I I I}}=-0.1266, \\
& \overline{\mu_{I V}}=-0.0652, \quad \overline{\mu_{V}}=-0.1135, \quad \overline{\mu_{V I}}=-0.1234 \text {. }
\end{aligned}
$$

It is easy to check that only the link $I I$ satisfies (20), i.e., $\mathcal{E}_{c}=\{I I\}$. The resulting partitions and the corresponding agents are presented in Figure 6a. Notice that $\mathbf{x}_{a_{1}}, \mathbf{x}_{a_{3}} \in \mathbb{R}^{2}$, $\mathbf{u}_{a_{1}}, \mathbf{u}_{a_{3}} \in \mathbb{R}$ and $\mathbf{x}_{a_{2}} \in \mathbb{R}^{4}, \mathbf{u}_{a_{2}} \in \mathbb{R}^{2}$.

Once we have the agents defined, we can check that there are two set of links connecting the same pairs of agents: $\{I, V\}$ for $a_{1}, a_{2}$ and $\{I I I, V I\}$ for $a_{2}, a_{3}$, from where we have to choose the cheapest one for each case. In this way, since

$$
\overline{\mu_{I}}<\overline{\mu_{V}}, \quad \overline{\mu_{I I I}}<\overline{\mu_{V I}},
$$

we remove, respectively, the redundant links $V$ and $V I$ and, consequently, $\mathcal{E}_{s}=\{V, V I\}$.

Finally, we need to compute whether there are any possible links between the agents that are costly enough to keep them always disconnected. Hence, according to (24) we can easily check that $\mathcal{E}_{e}=\{I V\}$. Therefore, the configuration is defined by the network $\left(\left\{a_{1}, a_{2}, a_{3}\right\},\{I, I I I\}\right)$ as it is shown in Figure $6 b$.

Let us consider a second scenario with the same dynamics given in (26) except the following difference:

$$
\mathbf{A}_{22}^{\prime}=\left[\begin{array}{cc}
1 & 0.9 \\
0 & -1.2
\end{array}\right]
$$




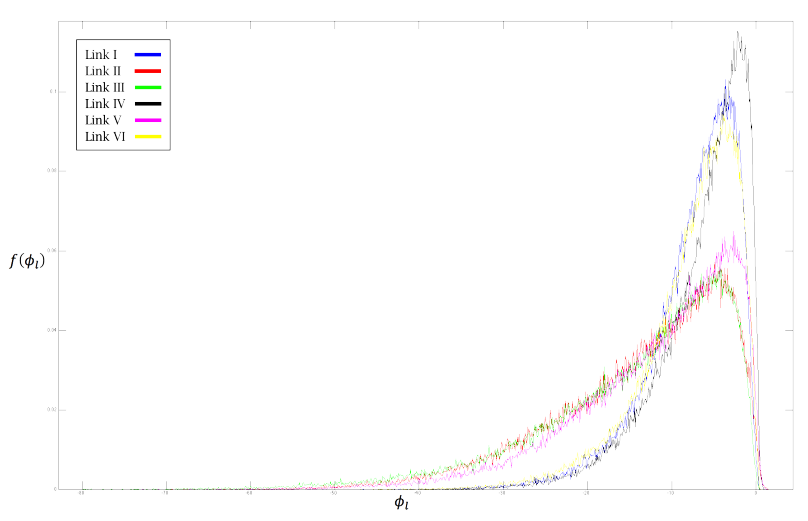

Fig. 7. Probability density function for the Shapley values: second scenario

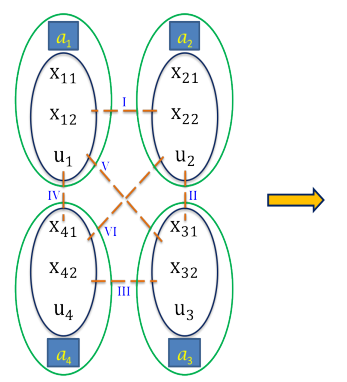

a)

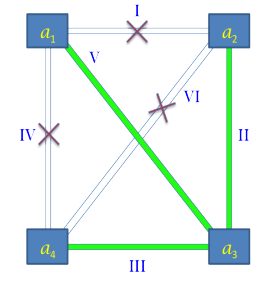

b)
Fig. 8. System partitioning: second scenario

and then apply again the partitioning algorithm. The probability density function for the Shapley value of each link in this case it is shown in Figure 7, where

$$
\begin{aligned}
& \overline{\mu_{I}}=-0.0928, \quad \overline{\mu_{I I}}=-0.1680, \quad \overline{\mu_{I I I}}=-0.1774, \\
& \overline{\mu_{I V}}=-0.0785, \quad \overline{\mu_{V}}=-0.1455, \quad \overline{\mu_{V I}}=-0.0969 .
\end{aligned}
$$

Now, there are no links that satisfy (20), i.e., $\mathcal{E}_{c}=\{\emptyset\}$. Hence, we have four agents as represented in Figure 8a and, according to (23), $\mathcal{E}_{s}=\{\emptyset\}$. Finally, the links $I, I V$ and $V I$ verify (24) so they will be kept disconnected. In this way, the configuration in this scenario is described by the network $\left(\left\{a_{1}, a_{2}, a_{3}, a_{4}\right\},\{I I, I I I, V\}\right)$ as illustrated in Figure 8b.

We conclude that the system partitioning has been achieved according to metrics proposed. Notice that the partitioning criterion can be relaxed or restricted depending on the chosen thresholds $\mathcal{L}_{c}$ and $\mathcal{L}_{e}$.

\section{CONCLUSIONS}

In this paper, we provide an offline method that, by using the Shapley value as a payoff rule, establishes the expected importance of the connections involved in the dynamics of a given system. These connections are interpreted as players of a cooperative game whose characteristic function depends on an upper bound on the cost-to-go of the closed-loop system. As the result of this method, a design algorithm for establishing the proper system partitioning that would merge/connect the different pieces of the system according to their degree of coupling is provided.

Future work will include the extension to nonequiprobable distributions for the states and the use of new metrics in this context. Likewise, the application to real largescale systems will be also addressed.

\section{REFERENCES}

[1] J. M. Maestre and R. R. Negenborn, Eds., Distributed Model Predictive Control Made Easy, ser. Intelligent Systems, Control and Automation: Science and Engineering. Springer, 2014, vol. 69.

[2] D. D. Siljak, Decentralized Control of Complex Systems. Boston, Massachusetts, USA: Academic Press, 1991.

[3] G. Fleischanderl and A. Haselboeck, Thoughts on Partitioning LargeScale Configuration Problems, AAAI Technical Report FS-96-03, 1996.

[4] N. Motee and B. Sayyar-Rodsari, "Optimal partitioning in distributed model predictive control," in Proceedings of the $21^{\text {th }}$ American Control Conference (ACC 2003), Denver, Colorado, USA, June 2003 pp. 5300-5305.

[5] D. Barcelli, C. Ocampo-Martínez, V. Puig, and A. Bemporad, "Decentralized model predictive control of drinking water networks using an automatic subsystem decomposition approach," in Proceedings of the $12^{\text {th }}$ IFAC Symposium on Large Scale Systems: Theory and Applications, Villeneuve d'Ascq, France, July 2010, pp. 572-577.

[6] C. Ocampo-Martínez, S. Bovo, and V. Puig, "Partitioning approach oriented to the decentralized predictive control of large-scale systems," Journal of Process Control, vol. 21, no. 5, pp. 775-786, June 2011.

[7] R. R. Negenborn, "Multi-agent model predictive control with applications to power networks," Ph.D. dissertation, Delft University of Technology, The Netherlands, December 2007.

[8] R. R. Negenborn, B. De Schutter, and J. Hellendoorn, "Multi-agent model predictive control for transportation networks: Serial versus parallel schemes," Engineering Applications of Artificial Intelligence, vol. 21, no. 3, pp. 353-366, April 2008.

[9] J. Anderson, Y. Chang, and A. Papachristodoulou, "Model decomposition and reduction tools for large-scale networks in systems biology," Automatica, vol. 47, no. 6, pp. 1165-1174, June 2011.

[10] Z. Zhou, S. Lin, and Y. Xi, "A dynamic network partition method for heterogenous urban traffic networks," in Proceedings of the $15^{\text {th }}$ International IEEE Conference on Intelligent Transportation Systems, Anchorage, Alaska, USA, September 2012, pp. 820-825.

[11] L. S. Shapley, "A value for $n$-person games," Contributions to the Theory of Games II. Edited by H. W. Kuhn and A. W. Tucker. Annals of Mathematics Studies, vol. 28, pp. 307-317, 1953.

[12] A. Nuñez, C. Ocampo-Martínez, B. De Schutter, F. Valencia, J. López, and J. Espinosa, "A multiobjective-based switching topology for hierarchical model predictive control applied to a hydro-power valley," in Proceedings of the $3^{\text {th }}$ IFAC International Conference on Intelligent Control and Automation Science (ICONS 2013), Chengdu, China, September 2013.

[13] J. M. Maestre, D. Muñoz de la Peña, A. Jiménez Losada, E. Algaba, and E. F. Camacho, "A coalitional control scheme with applications to cooperative game theory," Optimal Control Applications and Methods, vol. 35, no. 5, pp. 592-608, September/October 2014.

[14] J. M. Maestre, D. Muñoz de la Peña, A. Jiménez Losada, E. Algaba, and E. F. Camacho, "An application of cooperative game theory to distributed control," in Proceedings of the $18^{\text {th }}$ IFAC World Congress, Milano, Italy, September 2011, pp. 9121-9126.

[15] J. M. Maestre, F. J. Muros, F. Fele, and E. F. Camacho, "An assessment of coalitional control in water systems," to be published in the Proceedings of the $14^{\text {th }}$ European Control Conference (ECC 2015), Linz, Austria, July 2015.

[16] F. J. Muros, J. M. Maestre, E. Algaba, T. Alamo, and E. F. Camacho, "Constraints on the Shapley value for a coalitional control system," in Proceedings of the $13^{\text {th }}$ European Control Conference (ECC 2014), Strasbourg, France, June 2014, pp. 1223-1228.

[17] F. J. Muros, J. M. Maestre, E. Algaba, T. Alamo, and E. F. Camacho, "An iterative design method for coalitional control networks with constraints on the Shapley value," in Proceedings of the $19^{\text {th }}$ IFAC World Congress, Cape Town, South Africa, August 2014, pp. 11881193.

[18] R. Fletcher and S. Leyffer, "Numerical experience with lower bounds for MIQP branch-and-bound," SIAM Journal on Optimization, vol. 8, no. 2, pp. 604-616, 1998.

[19] P. Gahinet, A. S. Nemirovskii, A. J. Laub, and M. Chilali, LMI Control Toolbox For Use with MATLAB ${ }^{\circledR}$, The MathWorks, Inc., 1995.

[20] G. Xu, T. S. H. Driessen, and H. Sun, "Matrix analysis for associated consistency in cooperative game theory," Linear Algebra and its Applications, vol. 428, no. 7, pp. 1571-1586, April 2008.

[21] G. Casella and R. L. Berger, Statistical Inference, $2^{\text {nd }}$ ed. Stamford, Connecticut, USA: Thomson Learning. Duxbury Advanced Ser., 2002. 